\title{
Phosphatidylinositol phosphates directly bind to neurofilament light chain (NF-L) for the regulation of NF-L self assembly
}

\author{
Sung-Kuk Kim¹, Ho Kim¹, Yong-Ryoul Yang ${ }^{2}$, \\ Pann-Ghill Suh ${ }^{2}$ and Jong-Soo Chang ${ }^{1,3}$ \\ ${ }^{1}$ Department of Life Science \\ College of Natural Science \\ Daejin University \\ Kyeonggido 487-711, Korea \\ ${ }^{2}$ School of Nano-Biotechnology and Chemical Engineering \\ Ulsan National Institute of Science and Technology \\ Ulsan 689-798, Korea \\ ${ }^{3}$ Corresponding author: Tel, +82-31-539-1853; \\ Fax, +82-31-539-1850; E-mail, jchang@ daejin.ac.kr \\ DOI 10.3858/emm.2011.43.3.019
}

Accepted 17 February 2011

Available Online 22 February 2011

Abbreviations: GST, glutathione S-transferase; NF-L, neurofilament light chain; $\mathrm{PH}$, pleckstrin homology; $\mathrm{PIP}_{2}$, phosphatidylinositol 4,5-bisphosphate; PtdlnsP, phosphatidylinositol phosphate; PLC- $\gamma 1$, phospholipase $\mathrm{C}-\gamma 1$

\begin{abstract}
Phosphatidylinositol phosphates (PtdlnsPs) are ubiquitous membrane phospholipids that play diverse roles in cell growth and differentiation. To clarify the regulation mechanism acting on neurofilament light chain (NF-L) self assembly, we examined the effects of various PtdlnsPs on this process. We found that PtdlnsPs, including $\mathrm{PI}(4,5) \mathrm{P}_{2}$, directly bind to the positively charged $\mathrm{Arg}^{54}$ of murine NF-L, and this binding promotes NF-L self assembly in vitro. Mutant NF-L (R53A/R54A) proteins lacking binding affinity to PtdlnsPs did not have the same effect, but the mutant NF-L proteins showed greater self assembly than the wild-type in the absence of any PtdlnsP. These results collectively suggest that $\mathrm{Arg}^{54}$ plays a pivotal role in NF-L self assembly by binding with PtdlnsPs.
\end{abstract}

Keywords: neurofilament protein L; phosphatidylinositol phosphates; phospholipase $\mathrm{C} \gamma$

\section{Introduction}

Phosphatidylinositol phosphates (PtdlnsPs) play multiple roles in cell growth and differentiation (Berridge, 1993; Nishizuka, 1995; Lemmon et al., 1996; Carlton and Cullen, 2005), acting as: (i) precursors of second messenger molecules; (ii) effectors for the localization and assembly of protein molecules; and (iii) components of the cellular plasma membrane.

Neurofilaments (NFs), the most abundant cytoskeletal components in large myelinated axons, are obligate heteropolymers composed of NF-L, NF-M and NF-H (Ching and Liem, 1993; Lee et al., 1993; Nakagawa et al., 1995). Each NF is composed of three domains: an $\alpha$-helix-rich rod domain in the middle, flanked by an $\mathrm{N}$-terminal head domain and a C-terminal non- $\alpha$-helical tail domain (Fuchs and Weber, 1994; Grant and Pant, 2000; Al-Chalabi and Miller, 2003). NF-L is known to self-assemble into core filaments that then form cross-bridges with NF-M or NF-H (Fuchs and Weber, 1994; Nakagawa et al., 1995). Recent studies have revealed that overexpression or point mutation of NF-L can result in abnormal NF assembly and aggregation in neuronal cell bodies (Cote et al., 1993; Xu et al., 1993; Mathieu et al., 1995; Carter et al., 1996; Lee et al., 2008). NF aggregations due to overexpression or point mutations of NF-L have been associated with neurodegenerative diseases such as Charcot-Marie-Tooth disease (Watson et al., 1994; Fabrizi et al., 2004), Alzheimer's disease (Shepherd et al., 2002; Norgren et al., 2003) and amyotrophic lateral sclerosis (Hirano et al., 1984; Collard et al., 1995; Al-Chalabi and Miller, 2003). Notably, phosphorylation of the NF-L head domain can inhibit self assembly or induce disassembly of polymerized NF-L (Sihag and Nixon, 1991; Guan et al., 1992; Hisanaga et al., 1994; Gibb et al., 1996; Mukai et al., 1996; Yates et al., 2009). However, the factors and mechanisms that regulate NF assembly are not yet fully understood. Thus, we need to identify and characterize additional factors capable of regulating the cytoplasmic assembly, axonal transport and organization of NFs. Phospholipase C- $\gamma 1$ (PLC- $\gamma 1$ ) plays a pivotal role in cell growth and differentiation by hydrolyzing phosphatidylinositol 4,5-bisphosphate $\left(\mathrm{PIP}_{2}\right)$ to inositol 1,4,5-trisphosphate $\left(I_{3}\right)$ and diacylglycerol (DG) 
A
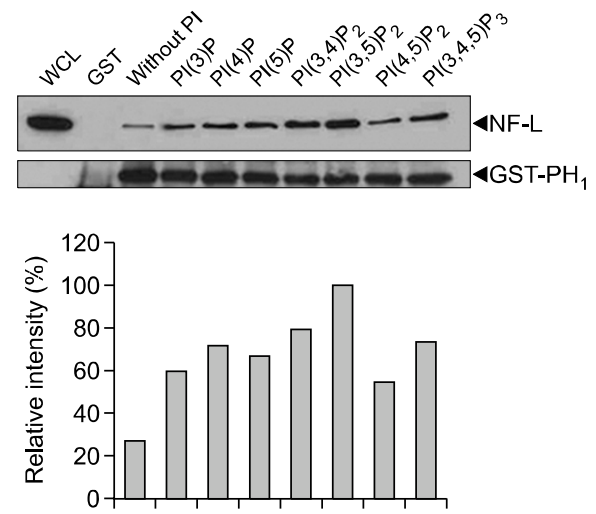

B

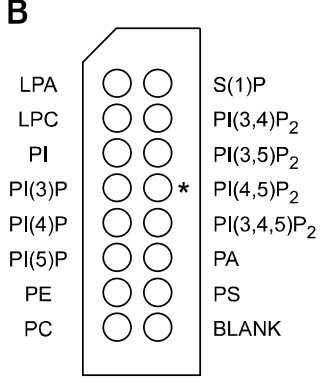

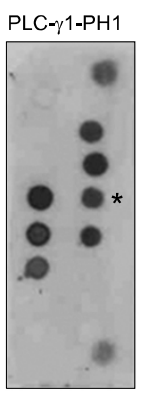

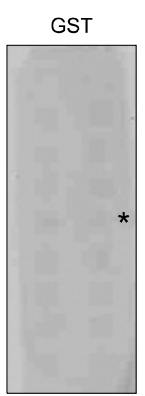

NF-L $(H)$

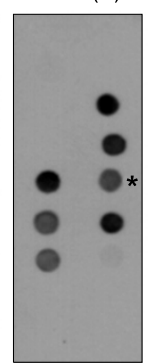

NF-L (R)

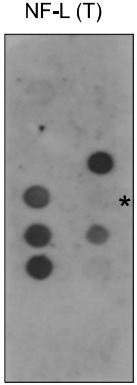

Figure 1. PtdlnsPs promote the interaction between the PH1 domain of PLC- $\gamma 1$ and NF-L. (A) GST-PLC- $\gamma 1-P H 1$ domain fusion proteins were incubated with NGF-treated PC12 cell extracts (NF-L pool) in the presence of different PtdlnsPs $(50 \mu \mathrm{g} / \mathrm{ml})$, and bound proteins were analyzed by Western blotting with anti-NF-L (upper) and anti-GST (middle) antibodies. The relative intensities of the NF-L bands were analyzed using image analysis software (Quantity One, Bio-Rad) (lower). Two independent experiments showed similar results. WCL represents whole cell lysate. (B) Dot-blot analysis shows that PtdlnsPs directly bind NF-L. PIP strips were incubated with purified GST, GST-PH1, GST-NF-L (H), GST-NF-L (R) and GST-NF-L (T) fusion proteins $(0.5 \mu \mathrm{g} / \mathrm{ml})$ in TBT buffer containing $2 \%$ non-fat skim milk for $14 \mathrm{~h}$ at $4^{\circ} \mathrm{C}$. After washes with TBT buffer, the membranes were incubated with a goat anti-GST antibody for $2 \mathrm{~h}$ at room temperature. Finally, the bound proteins were detected with an HRP-conjugated anti-goat antibody. Each dot contained 100 pM of PI. LPA, lysophosphatidic acid; LPC, lysophosphocholine; PI, phosphatidylinositol; PE, phosphatidylethanolamine; PC, phosphatidylcholine; S(1)P, sphingosine-1-phosphate; $\mathrm{PA}$, phosphatidic acid; PS, phosphatidylserine. The asterisk (*) represents the position of $\mathrm{PI}(4,5) \mathrm{P}_{2}$.

(Suh et al., 1988; Berridge, 1993; Rhee, 2001; Shin et al., 2002). PLC- $\gamma 1$ has two pleckstrin homology $(\mathrm{PH})$ domains for protein-protein and proteinlipid interactions (Gibson et al., 1994; Falasca et al., 1998; Chung et al., 2010): PH1 is located in the 150 amino acid residues closest to the $\mathrm{N}$-terminus, while $\mathrm{PH} 2$ is located near the center of the molecule and is split by an $\mathrm{SH} 2-\mathrm{SH}_{2}-\mathrm{SH} 3$ domain (Gibson et al., 1994; Chang et al., 2002). PH domains bind with high specificity and affinity to phosphatidylinositol phosphates (PtdlnsPs) such as $\mathrm{PI}(3) \mathrm{P}, \mathrm{PI}(4) \mathrm{P}, \mathrm{PI}(5) \mathrm{P}, \mathrm{PI}(3,4) \mathrm{P}_{2}, \mathrm{PI}(3,5) \mathrm{P}_{2}$, $\mathrm{PI}(4,5) \mathrm{P}_{2}$ and $\mathrm{PI}(3,4,5) \mathrm{P}_{3}$ (Lemmon and Ferguson, 2000; Cozier et al., 2004; Carlton and Cullen, 2005; Balla, 2005). During our study of the interaction between the $\mathrm{PH}$ domains of PLC- $\gamma 1$ and NF-L (Kim et al., 2006), we found that NF-L also associates with PtdlnsPs.

Here, we show for the first time that NF-L directly binds PtdlnsPs to regulate NF-L self assembly. These findings provide important new insights into the roles of PtdlnsPs in neuronal differentiation.

\section{Results}

\section{PtdlnsPs promote the association between NF-L and the PH1 domain of PLC- $\gamma 1$}

In our studies aimed at understanding the relationship between NF-L and PLC- $\gamma 1$-dependent signaling, we found that the PH1 domain of PLC- $\gamma 1$ directly binds to NF-L (Kim et al., 2006). Because the $\mathrm{PH}$ domain is known to be a PtdlnsP-binding motif in numerous proteins (Lemmon and Ferguson, 1996; Klopfenstein et al., 2002; Klopfenstein and Vale, 2004), we examined whether PtdlnsPs could be involved in the molecular interaction between the PLC- $\gamma 1 \mathrm{PH} 1$ domain and NF-L. We performed a GST-PLC- $\gamma 1-\mathrm{PH} 1$ pull-down assay using nerve growth factor (NGF)-treated PC12 cell lysates containing different PtdlnsPs in vesicle form including $\mathrm{PI}(3) \mathrm{P}, \mathrm{PI}(4) \mathrm{P}, \mathrm{PI}(5) \mathrm{P}, \mathrm{PI}(3,4) \mathrm{P}_{2}$, $\mathrm{PI}(3,5) \mathrm{P}_{2}, \mathrm{PI}(4,5) \mathrm{P}_{2}$ and $\mathrm{PI}(3,4,5) \mathrm{P}_{3}$. As shown in Figure $1 \mathrm{~A}$, substantially more NF-L proteins bound to the GST-PLC- $\gamma 1-\mathrm{PH} 1$ domain in PtdlnsP-harboring cells compared to untreated controls. This result led us to examine whether NF-L itself can bind 
A

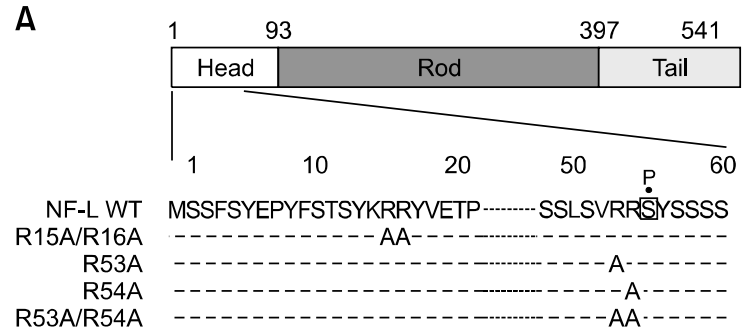

B

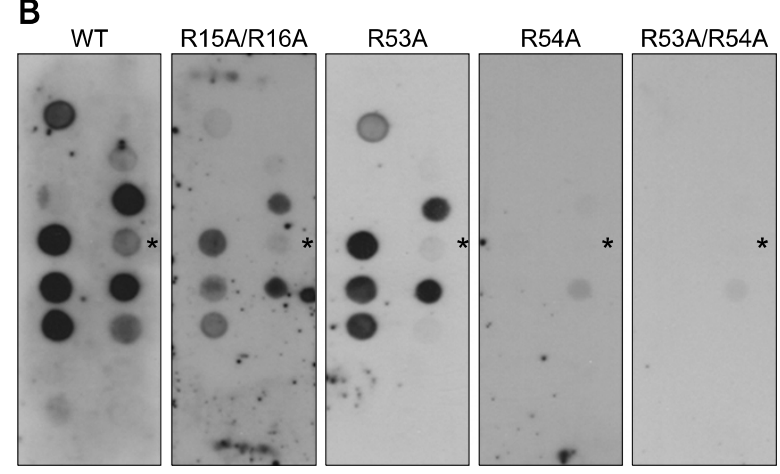

Figure 2. PtdlnsPs directly binds to $\mathrm{Arg}^{54}$ of NF-L. (A) Schematic representation of the NF-L head domain containing point mutations in arginine residues, which were replaced with the neutral amino acid, alanine. The boxed "S" represents a possible phospho-Ser. (B) Two site-directed point mutants of NF-L, R54A and R53A/R54A, had no binding affinity for PtdlnsPs. Point-mutant GST-fusion proteins were used for dot-blotting as described in Figure $1(\mathrm{~B})$. The asterisk (*) represents the position of $\mathrm{PI}(4,5) \mathrm{P}_{2}$.

PtdlnsPs.

\section{PtdlnsPs directly bind to NF-L}

To examine the binding affinity and specificity of NF-L for phospholipids, we purified GST-fused NF-L proteins containing GST-NF-L(H), -NF-L(R) and $-N F-L(T)$, which represent head $(H)$, rod $(R)$ and tail $(T)$ domains of NF-L, from an $E$. coli expression system and applied the recombinant proteins to dot-blot analysis using Echelon strips (See 'Methods'). Both the NF-L(H) and NF-L(T) domains specifically bound all of the tested PtdlnsPs with high affinity (Figure 1B), whereas the NF-L(R) domain showed a weak affinity for only $\mathrm{PI}(3,4,5) \mathrm{P}_{3}$. Thus, NF-L directly binds to phospholipids containing the phosphatidylinositol rings of PtdlnsPs but not to other tested lipids, such as phosphataidylinositol (PI), phosphatidylethanol (PE), phosphatidylcholine (PC) and sphingosine 1phosphate (S(1)P) (Figure 1B). NF-L(H) and NF-L(T) showed the highest affinity for $\mathrm{PI}(3,5) \mathrm{P}_{2}$, which strongly promoted the molecular interaction between NF-L and the PLC- $\gamma 1 \mathrm{PH} 1$ domain (Figure 1A). NF-L(H) and NF-L(T) showed the lowest affinity to $\mathrm{PI}(4,5) \mathrm{P}_{2}$, which only moderately promoted

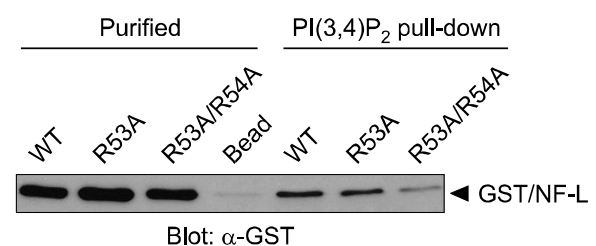

Figure 3. The R53A/R54A mutant shows reduced binding affinity for $\mathrm{PIP}_{2}$. Bacterially expressed GST-NF-L proteins were incubated with $\mathrm{PIP}_{2}$ bound agarose beads for $1 \mathrm{~h}$ at $4^{\circ} \mathrm{C}$, and the bound proteins were quantified by Western blotting.

the interaction between NF-L and the PLC- $\gamma 1$ PH1 domain (Figure $1 \mathrm{~A}$ ). The PLC- $\gamma 1 \mathrm{PH} 1$ domain also showed high affinities for $\mathrm{PI}(3,5) \mathrm{P}_{2}$ and $\mathrm{PI}(3) \mathrm{P}$, and had a higher affinity to $\mathrm{PI}(4,5) \mathrm{P}_{2}$ than did the NF-L protein (Figure 1B).

Since our results revealed that the NF-L molecule binds to PtdlnsPs but not to other phospholipids (e.g., $\mathrm{PI}, \mathrm{PC}$ or $\mathrm{PE}$ ), we speculated that the negatively charged phosphate groups in $3^{\text {rd }}, 4^{\text {th }}$ and $5^{\text {th }}$ carbon positions of the inositol ring may supply the binding affinity to NF-L. If this is true, then the positively charged basic amino acid residues of NF-L may be involved in binding the phosphate group of the inositol ring. To determine the amino acid residue(s) in NF-L responsible for binding PtdlnsPs, we constructed point mutants in which $\mathrm{Arg}^{15} / \mathrm{Arg}^{16}$, $\mathrm{Arg}^{53}, \mathrm{Arg}^{54}$ and $\mathrm{Arg}^{53} / \mathrm{Arg}^{54}$, one or two basic amino acids in the head domain of NF-L, were replaced with alanine (Ala; a neutral amino acid) (Figure 2A). As shown in Figure 2B, the R54A and R53A/R54A mutants showed almost no affinity for the tested PtdlnsPs (Figure 2B), while the R53A and R15A/R16A mutants showed only slight decreases in their binding affinities for the tested PtdlnsPs (Figure 2B). These results suggest that the positively charged $\mathrm{Arg}^{54}$ is responsible for the binding of NF-L with PtdlnsPs. The Ser ${ }^{55}$ residue ( $S$; boxed in Figure 2A) may be phosphorylated by some protein kinases (Guan et al., 1992; Gibb et al., 1996; Grant and Pant, 2000).

We also assessed the $\mathrm{PIP}_{2}$ binding affinity for R53A/R54A mutant and wild-type NF-L using PIP-bound agarose beads (Figure 3 ). The beads were incubated with GST-R53A, -R53A/R54A or -wild-type NF-L for $1 \mathrm{~h}$ at $4^{\circ} \mathrm{C}$, and then extensively washed with buffer. As shown in Figure 3, R53A/ R54A mutant NF-L showed a substantially reduced affinity for PIP2 compared with that of wild-type NF-L $(H)$, suggesting that the head domain of NF-L lost its affinity for $\mathrm{PIP}_{2}$ upon mutation of $\mathrm{Arg}^{54}$ residue. In addition to NF-L head domain, there are so many basic amino acid residues in the tail domain of NF-L that shows a high affinity for PtdlnsPs (Figure 1B). We then tried to identify the 

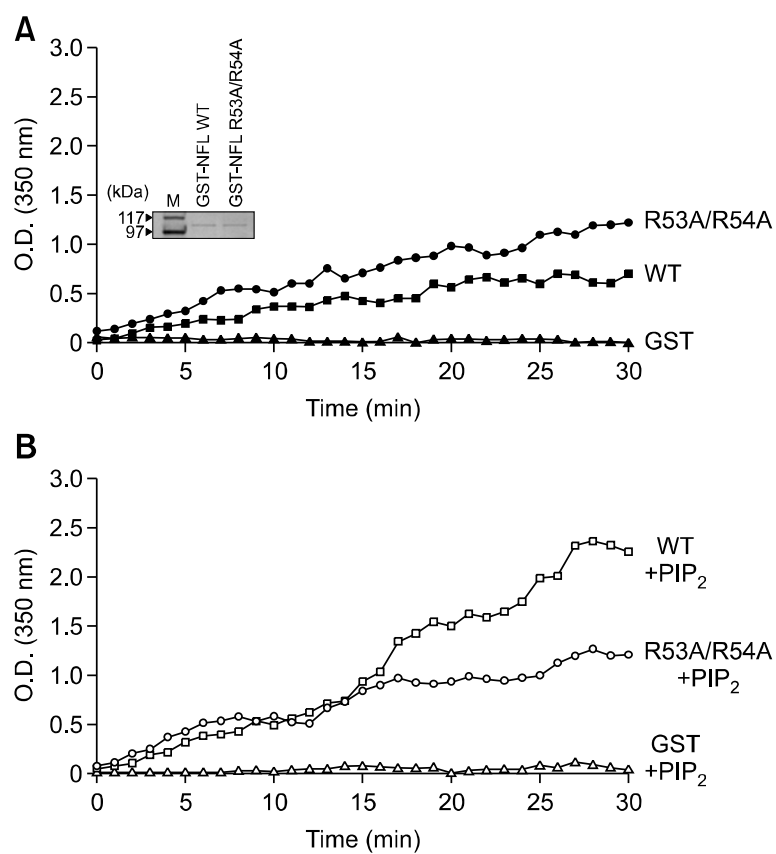

Figure 4. PtdlnsP promotes NF-L assembly by direct binding to the $\mathrm{Arg}^{54}$ of NF-L. (A) Comparison of NF-L self assembly between wild-type and R53A/R54A mutant NF-L in vitro. Bacterially expressed GST-NF-L proteins were incubated in NF-L polymerizing buffer and the optical density (OD) was measured over time. (B) PIP 2 affects NF-L self assembly. Wild-type and R53A/R54A mutant NF-L were incubated in the presence of $\mathrm{PIP}_{2}$, and the OD was measured over time. Wild-type NF-L self assembly was promoted by $\mathrm{PIP}_{2}$, whereas that of the mutant (which did not bind $\mathrm{PIP}_{2}$ ) was not.

binding partner residues of PtdlnsPs within the tail domain, none of 10 point mutants (substitutes alanine for arginine or lysine residue) showed decreased binding affinity for PtdlnsPs. So we used only head domain mutant, NF-L(H)-R53A/ R54A, for further experiments.

\section{PIP $_{\mathbf{2}}$ accelerates NF-L self assembly}

Since purified NF-L can self assemble in vitro (Hisanaga and Hirokawa, 1990; Lee et al., 1993), we next compared NF-L self assembly between R53A/R54A mutant and wild-type NF-L using the GST-fusion proteins. As shown in Figure 4A, R53A/ R54A mutant NF-L showed higher self-assembly activity than wild-type NF-L, whereas the control GST protein showed basal activity (Figure 4A). Next, we compared the levels of NF-L self assembly between wild-type GST-NF-L and GST- NF-L-R53A/ R54A mutant in the presence of PtdlnsPs. The self-assembly reaction of the GST- NF-L-R53A/ R54A mutant was unchanged in the presence of $\mathrm{PIP}_{2}$, whereas that of wild-type NF-L was greatly increased by the presence of $\mathrm{PIP}_{2}$ (Figure 4B), suggesting that binding of $\mathrm{PIP}_{2}$ accelerates NF-L
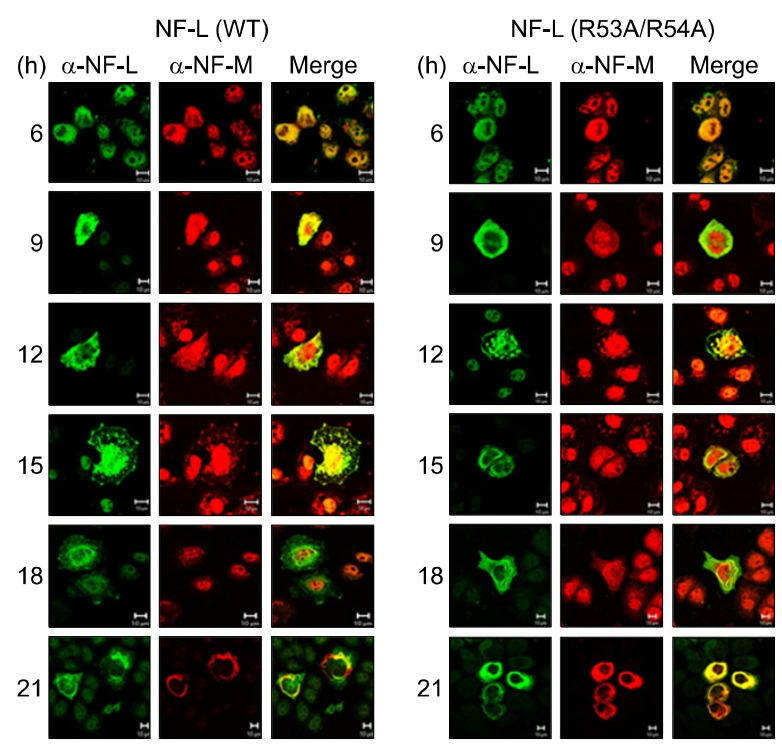

Figure 5. The R53A/R54A mutant shows accelerated NF assembly in intact cells. The cDNAs encoding rat NF-M/NF-L (wild set, left) or NF-M/NF-L-R53A/R54A (mutant set, right) were transiently co-transfected into SW13 cells ( $\mathrm{Vim}^{-}$) grown on glass coverslips. The cells were fixed in $4 \%$ paraformaldehyde at the indicated times, and subjected to immunostaining with anti-NF-L and anti-NF-M antibodies, followed by detection using FITC- and rhodamine-conjugated secondary antibodies, respectively.

self assembly in vitro. We tested other PtdlnsPs that showed binding to the NF-L head domain (Figure 1B), and found that their self-assemblypromoting effects on NF-L were similar to those of $\mathrm{PIP}_{2}$ (data not shown).

\section{The R53A/R54A mutant shows accelerated NF-L assembly in vivo}

Finally, we compared the level of filament formation between the mutant and wild-type NF-L proteins in intact cells. The vimentin-deficient human adrenal carcinoma-derived SW13 cell line has been used for the examination of intermediate- filament formation (Lee et al., 1993). Here, we examined the filament formation levels in SW13 cells co-transfected with vectors encoding rat NF-M with either wild-type NF-L (wild set) or NF-L-R53A/ R54A (mutant set). The transfected cells were fixed and double stained with anti-NF-M and antiNF-L antibodies at various time points. As shown in Figure 5, SW13 cells transfected with the mutant formed intermediate filaments within $15 \mathrm{~h}$ after transfection, whereas cells transfected with the wild-type construct needed at least $18 \mathrm{~h}$ to form filaments. The mutant set also developed rod-like thick filaments over time, while wild set showed thread-like filaments (Figure 5). This result suggests that in vivo intermediate-filament formation by NF-L 
is regulated by binding between PtdlnsPs and the $\mathrm{Arg}^{54}$ residue.

\section{Discussion}

We herein show that the PtdlnsPs directly interact with NF-L and regulate neuronal differentiation by binding with the NF-L head domain along with the $\mathrm{PH} 1$ domain of PLC- $\gamma 1$. We also examined whether PtdlnsPs affect NF polymerization, and show for the first time that a number of PtdlnsPs, including $\mathrm{PI}(3) \mathrm{P}, \mathrm{PI}(4) \mathrm{P}, \mathrm{PI}(5) \mathrm{P}, \mathrm{PI}(3,4) \mathrm{P}_{2}, \mathrm{PI}(3,5) \mathrm{P}_{2}$ and $\mathrm{PI}(3,4,5) \mathrm{P}_{3}$, directly bind NF-L molecules (Figure 1B). All of the tested PtdlnsPs bound the NF-L head and tail domains with different affinities, and were found to promote NF-L self assembly in vitro. Interestingly, among all tested PtdlnsPs, $\mathrm{PI}(4,5) \mathrm{P}_{2}$ showed the poorest binding to NF-L.

Previously, researchers had hypothesized that charge-dependent polymerization (phosphorylation/ dephosphorylation) of the head domain of NF-L is responsible for regulating NF-L self assembly (Sihag and Nixon, 1991; Hisanaga et al., 1994; Nakamura et al., 2000; Yates, 2009). The head domain of mammalian NF-L has an Arg-Arg-Ser (RRS) sequence (amino acid residues 53-55), where $S^{55}$ can be phosphorylated by protein kinases to preclude NF-L self assembly (Sihag and Nixon, 1991; Nakamura et al., 2000; Yates et al., 2009). These previous findings seem to indicate that binding of the negatively charged phosphate group from residue $\mathrm{Ser}^{55}$ of the NF-L head domain precludes self assembly (Gibb et al., 1996; Grant and Pant, 2000; Yates et al., 2009).

Interestingly, our present findings suggests a new regulation mechanism wherein the positively charged $\mathrm{Arg}^{54}$ acts as an adaptor for PtdlnsPs, which function as positive effectors for NF-L assembly. Consistent with this hypothesis, we found that the R53A/R54A double mutant was resistant to PtdlnsPinduced NF-L self assembly. However, in the absence of any PtdlnsP, the R53A/R54A mutant was more efficient than the wild-type protein for self assembly both in vitro (Figure 4) and in vivo, as assessed by intermediate-filament formation in SW13 cells (Figure 5). The mutant-transfected SW13 cells showed a characteristic pattern of filament assembly, characterized by rapid self assembly and the formation of thick, rod-like filaments (Figure 5). Our observations that replacement of the positively charged arginine with a neutral amino acid increased the efficiency of NF-L self assembly may suggest that the positive charge of $\mathrm{Arg}^{54}$ attenuates the self assembly of wild-type NF-L.

In sum, our present findings suggest that $\mathrm{Arg}^{54}$ plays a key role in NF-L self assembly and neurofilament formation, likely via an association with PtdlnsPs and/or the direct attenuation of NF-L self assembly. These observations provide important new insights into the roles of PtdlnsPs in neuronal differentiation.

\section{Methods}

\section{Antibodies and cell culture}

Monoclonal anti-NF-L (mAb 1615) and polyclonal anti-NF-L were purchased from Chemicon (Temecula, CA). Monoclonal anti-NF-M (mAb RNF403) was purchased from MP Biochemicals (Aurora, OH). The horseradish peroxidase (HRP)-conjugated goat anti-mouse and goat anti-rabbit antibodies were purchased from Upstate Biotechology Inc. (Lake Placid, NY). Fluorescein-conjugated Affinipure goat anti-rabbit $\lg$ and rhodamine-conjugated Affinipure goat anti-mouse IgG were from Jackson ImmunoResearch Laboratories (West Grove, PA). All PtdlnsPs were purchased from Sigma-Aldrich (St. Louis, MO), and the Echelon Strips (P-6001) and PIP-bound agarose beads (P-B045a) were purchased from Echelon Bioscience Inc. (Salt Lake City, UT).

PC12 cells were cultured at $37^{\circ} \mathrm{C}$ in Dulbecco's modified Eagle's medium (DMEM) containing $10 \%$ fetal bovine serum (FBS), $5 \%$ horse serum and $100 \mathrm{U} / \mathrm{ml}$ penicillinstreptomycin. The human adrenal carcinoma SW13 cell line $\left(\mathrm{Vim}^{-}\right)$was grown at $37^{\circ} \mathrm{C}$ in DMEM supplemented with $10 \%$ FBS.

\section{Vector constructs and protein expression}

For expression in $E$. coli, the cDNA sequence encoding the amino-terminal $\mathrm{PH}$ domain (PH1) of rat PLC- $\gamma 1$ (amino acids 25-145) (Suh et al., 1988) was ligated into the pGEX-5X-1 vector (Amersham Pharmacia Biotech Inc. Piscataway, NJ) for expression of a GST-PH1 fusion protein, as previously described (Chang et al., 2002). PCRamplified rat cDNAs encoding NF-L (purchased from the American Type Culture Collection, Manassas, VA) were ligated into the EcoRI/Sall restriction site of pGEX-5X-1. For the expression of domain-specific NF-L fusion proteins, PCR-amplified cDNAs for the NF-L head domain [NF-L(H), amino acid residues 1-93], rod domain [NF-L(R), amino acid residues 93-397], head/rod domain [NF-L(H/R), amino acid residues 1-397], and tail domain [NF-L(T), amino acid residues 398-542] were separately inserted into the EcoRI/Sall sites of pGEX-5X-1. The GST-NF-L-R54A and GST-NF-L-R53A/R54A point mutants were engineered by PCR-based point mutation (Takara Bio Inc. Kyoto, Japan). All constructs were prepared using a Qiagen Plasmid Maxi Kit (Qiagen Inc., Santa Clarita, CA) and confirmed by direct sequencing of the ligation and mutation sites.

The GST fusion proteins were expressed in E. coli, and lysates were incubated with glutathione sepharose (GSH) beads. The bound beads were washed extensively with Igepal buffer $[20 \mathrm{mM}$ Tris-Cl, pH 7.5, 1\% Igepal CA-630, $300 \mathrm{mM} \mathrm{NaCl}, 2 \mathrm{mM} \mathrm{MgCl}$, $1 \mathrm{mM}$ EDTA, $10 \mu \mathrm{g} / \mathrm{ml}$ aprotinin, $10 \mu \mathrm{g} / \mathrm{ml}$ leupeptin, $1 \mathrm{mM}$ phenylmethylsulfonyl fluoride 
(PMSF) and $1 \mathrm{mM}$ sodium orthovanadate], and the purified proteins were eluted with a buffer containing reduced glutathione.

To examine intermediate-filament formation in SW13 cells, cDNAs encoding rat NF-L (purchased from ATCC) and NF-M (kindly provided by Dr. S. Hisanaga, Tokyo Metropolitan University, with the permission of Dr. R. K. H. Liem, Columbia University) were ligated into the pCMVFLAG (Clontech Laboratories, Mountain View, CA) and pREFA vectors (Chang et al., 1997), respectively. The resulting constructs, pFLAG-NF-L and pREFA-NF-M, produced proteins that were FLAG-tagged at their N-termini. SW13 cells were transiently co-transfected with pFLAGNF-L and pREFA-NF-M using a ProFection kit (Promega, Madison, WI), which applies the calcium-phosphate coprecipitation method. The cells were fixed at $37^{\circ} \mathrm{C}$ for 10 min in $4 \%$ paraformaldehyde for either immunostaining using anti-NF-L and anti-NF-M antibodies, or immunoblotting with an anti-FLAG antibody. For green fluorescent protein (GFP)-fused NF-L expression, PC12 cells were transiently transfected with constructs encoding GFP-NF-L or GFP-NF-L-R53A/R54A fusion proteins, using a ProFection kit.

\section{Immunofluorescent microscopy}

SW13 cells were seeded on glass coverslips in 6-well plates, transfected using a ProFection kit, and grown for 2 days in DMEM. The cells were fixed at $37^{\circ} \mathrm{C}$ for $10 \mathrm{~min}$ in $4 \%$ paraformaldehyde, and then incubated with affinity purified monoclonal anti-NF-M or polyclonal anti-NF-L antibodies for $1 \mathrm{~h}$ at room temperature in a humidified chamber. Following complete washing with PBS, the cells were incubated with fluorescein-conjugated Affinipure goat anti-rabbit IgG or rhodamine-conjugated Affinipure goat anti-mouse IgG. Immunostained cells were photographed under a confocal laser-beam fluorescent microscope (PerkinElmer Inc., Waltham, MA).

\section{Dot-blot analysis}

The ability of the PH1 domain and NF-L proteins to bind different phospholipids was examined by dot-blot analysis using Echelon P-6001 strips (Echelon Bioscience Inc.). Each membrane was blocked with TBT buffer for $1 \mathrm{~h}$ and then incubated with purified GST, GST-PH1, GST-NF-L or point-mutant GST-NF-L fusion proteins $(0.5 \mu \mathrm{g} / \mathrm{ml})$ in TBT buffer containing $2 \%$ non-fat skim milk for $14 \mathrm{~h}$ at $4^{\circ} \mathrm{C}$. After washes with TBT buffer, the membranes were incubated with goat anti-GST antibodies for $2 \mathrm{~h}$ at room temperature. The membranes were then washed extensively with TBT buffer, and then the bound proteins were detected with HRP-conjugated anti-goat antibodies and visualized using an ECL detection system.

\section{In vitro assay of NF-L self-assembly}

To assess NF-L polymerization in vitro, purified GST-NF-L or mutant NF-L fusion proteins obtained from $E$. coli were dissolved in $200 \mu \mathrm{l}$ of NF-L polymerizing buffer $(20 \mathrm{mM}$ Tris-Cl, pH 7.0, $1 \mathrm{mM}$ dithiothreitol, $1 \mathrm{mM} \mathrm{MgCl}, 10 \mu \mathrm{g} / \mathrm{ml}$ leupeptin, $10 \mu \mathrm{g} / \mathrm{ml}$ aprotinin, $2 \mu \mathrm{g}$ GST-NF-L) and incu- bated for $0.5 \mathrm{~h}$ at $35^{\circ} \mathrm{C}$, and then optical density was measured at $350 \mathrm{~nm}$. To examine the effect of PtdlnsPs on NF-L self assembly, appropriate amounts of phospholipid vesicles (Li et al., 2006) were added before incubation and optical density was measured at $350 \mathrm{~nm}$ over time.

\section{Acknowledgements}

This work was supported by grants from the National Research Foundation of Korea (314-2008-1-C00294).

\section{References}

Al-Chalabi A, Miller CC. Neurofilaments and neurological disease. Bioessays 2003;25:346-55

Balla T. Inositol-lipid binding motifs: signal integrators through protein-lipid and protein-protein interactions. J Cell Sci 2005;118:2093-104

Berridge MJ. Inositol trisphosphate and calcium signalling. Nature 1993;361:315-25

Carlton JG, Cullen PJ. Coincidence detection in phosphoinositide signaling. Trends Cell Biol 2005;15:540-7

Carter JE, Gallo JM, Anderson VE, Anderton BH, Robertson $\mathrm{J}$. Aggregation of neurofilaments in NF-L transfected neuronal cells: regeneration of the filamentous network by a protein kinase C inhibitor. J Neurochem 1996;67:1997-2004

Chang JS, Noh DY, Park IA, Kim MJ, Song H, Ryu SH, Suh $\mathrm{P}-\mathrm{G}$. Overexpression of phospholipase C-gamma1 in rat $3 Y 1$ fibroblast cells leads to malignant transformation. Cancer Res 1997;57:5465-8

Chang JS, Seok H, Kwon T-K, Min DS, Ahn BH, Lee YH, Suh JW, Kim JW, Iwashita S, Omori A, Ichinose S, Numata O, Seo J-K, Oh YS, Suh P-G. Interaction of elongation factor-1alpha and pleckstrin homology domain of phospholipase Cgamma 1 with activating its activity. J Biol Chem 2002; 277:19697-702

Ching GY, Liem RK. Assembly of type IV neuronal intermediate filaments in nonneuronal cells in the absence of preexisting cytoplasmic intermediate filaments. J Cell Biol 1993;122:1323-35

Chung SH, Kim S-K, Kim JK, Yang Y-R, Suh P-H, Chang J-S. A double point mutation in PLC-gamma1 (Y509A/F510A) enhances $Y 783$ phosphorylation and inositol phospholipidhydrolyzing activity upon EGF stimulation. Exp Mol Med 2010;42:216-27

Collard JF, Cote F, Julien JP. Defective axonal transport in a transgenic mouse model of amyotrophic lateral sclerosis. Nature 1995;375:61-4

Cote F, Collard JF, Julien JP. Progressive neuronopathy in transgenic mice expressing the human neurofilament heavy gene: a mouse model of amyotrophic lateral sclerosis. Cell 1993;73:35-46

Cozier GE, Carlton J, Bouyoucef D, Cullen PJ. Membrane targeting by pleckstrin homology domains. Curr Top Microbiol Immunol 2004;282:49-88 
Fabrizi GM, Cavallaro T, Angiari C, Bertolasi L, Cabrini I, Ferrarini M, Rizzuto N. Giant axon and neurofilament accumulation in Charcot-Marie-Tooth disease type 2E. Neurology 2004;62:1429-31

Falasca M, Logan SK, Lehto VP, Baccante G, Lemmon MA, Schlessinger J. Activation of phospholipase $\mathrm{C}$ gamma by $\mathrm{PI}$ 3-kinase-induced $\mathrm{PH}$ domain-mediated membrane targeting. EMBO J 1998;17:414-22

Fuchs E, Weber K. Intermediate filaments: structure, dynamics, function, and disease. Annu Rev Biochem 1994;63: 345-82

Gibb BJ, Robertson J, Miller CC. Assembly properties of neurofilament light chain $\mathrm{Ser}^{55}$ mutants in transfected mammalian cells. J Neurochem 1996;66:1306-11

Gibson TJ, Hyvonen M, Musacchio A, Saraste M, Birney E. $\mathrm{PH}$ domain: the first anniversary. Trends Biochem Sci 1994;19:349-53

Grant P, Pant HC. Neurofilament protein synthesis and phosphorylation. J Neurocytol 2000;29:843-72

Guan RJ, Hall FL, Cohlberg JA. Proline-directed protein kinase (p34cdc2/p58cyclinA) phosphorylates bovine neurofilaments. J Neurochem 1992;58:1365-71

Hirano A, Donnenfeld H, Sasaki S, Nakano I. Fine structural observations of neurofilamentous changes in amyotrophic lateral sclerosis. J Neuropathol Exp Neurol 1984;43:461-70

Hisanaga S, Hirokawa N. Molecular architecture of the neurofilament. II. Reassembly process of neurofilament $\mathrm{L}$ protein in vitro. J Mol Biol 1990;211:871-82

Hisanaga S, Matsuoka Y, Nishizawa K, Saito T, Inagaki M, Hirokawa N. Phosphorylation of native and reassembled neurofilaments composed of NF-L, NF-M, and NF-H by the catalytic subunit of cAMP-dependent protein kinase. Mol Biol Cell 1994;5:161-72

Kim SK, Choi JH, Suh P-G, Chang J-S. Pleckstrin homology domain of phospholipase C-gamma1 directly binds to 68-kDa neurofilament light chain. Exp Mol Med 2006;38: 265-72

Klopfenstein DR, Tomishige M, Stuurman N, Vale RD. Role of phosphatidylinositol $(4,5)$ bisphosphate organization in membrane transport by the Unc104 kinesin motor. Cell 2002;109:347-58

Klopfenstein DR, Vale RD. The lipid binding pleckstrin homology domain in UNC-104 kinesin is necessary for synaptic vesicle transport in Caenorhabditis elegans. Mol Biol Cell 2004;15:3729-39

Lee IB, Kim S-K, Chung S-H, Kim H, Kwon T-K, Min DS, Chang J-S. The effect of rod domain A148V mutation of neurofilament light chain on filament formation. BMB Rep 2008;41:868-74

Lee MK, Xu Z, Wong PC, Cleveland DW. Neurofilaments are obligate heteropolymers in vivo. J Cell Biol 1993;122: 1337-50

Lemmon MA, Ferguson KM, Schlessinger J. PH domains: diverse sequences with a common fold recruit signaling molecules to the cell surface. Cell 1996;85:621-4
Lemmon MA, Ferguson KM. Signal-dependent membrane targeting by pleckstrin homology $(\mathrm{PH})$ domains. Biochem $\mathrm{J}$ 2000;350:1-18

Li L, Shin OH, Rhee JS, Araç D, Rah JC, Rizo J, Südhof T, Rosenmund C. Phosphatidylinositol phosphates as coactivators of $\mathrm{Ca}^{2+}$ binding to $\mathrm{C} 2$ domain of synaptotagmin 1 . J Biol Chem 2006;281:15845-52

Mathieu JF, Ma D, Descarries L, Vallee A, Parent A, Julien JP, Doucet G. CNS distribution and overexpression of neurofilament light proteins (NF-L) in mice transgenic for the human NF-L: aberrant accumulation in thalamic perikarya. Exp Neurol 1995;132:134-46

Mukai H, Toshimori M, Shibata H, Kitagawa M, Shimakawa $M$, Miyahara M, Sunakawa H, Ono Y. PKN associates and phosphorylates the head-rod domain of neurofilament protein. J Biol Chem 1996;271:9816-22

Nakagawa T, Chen J, Zhang Z, Kanai Y, Hirokawa N. Two distinct functions of the carboxyl-terminal tail domain of NF-M upon neurofilament assembly: cross-bridge formation and longitudinal elongation of filaments. J Cell Biol 1995;129: 411-29

Nakamura Y, Hashimoto R, Kashiwagi Y, Aimoto S, Fukusho E, Matsumoto N, Kudo T, Takeda M. Major phosphorylation site (Ser55) of neurofilament $L$ by cyclic AMP-dependent protein kinase in rat primary neuronal culture. J Neurochem 2000;74:949-59

Nishizuka Y. Protein kinase $C$ and lipid signaling for sustained cellular responses. FASEB J 1995;9:484-96

Norgren N, Rosengren L, Stigbrand T. Elevated neurofilament levels in neurological diseases. Brain Res 2003;987: 25-31

Rhee SG. Regulation of phosphoinositide-specific phospholipase C. Annu Rev Biochem 2001;70:281-312

Shepherd CE, McCann H, Thiel E, Halliday GM. Neurofilament-immunoreactive neurons in Alzheimer's disease and dementia with Lewy bodies. Neurobiol Dis 2002;9: 249-57

Shin SY, Yoon SC, Kim YH, Kim YS, Lee YH. Phosphorylation of glycogen synthase kinase-3beta at serine-9 by phospholipase Cgamma1 through protein kinase $\mathrm{C}$ in rat 3Y1 fibroblasts. Exp Mol Med 2002;34:444-50

Sihag RK, Nixon RA. Identification of Ser-55 as a major protein kinase A phosphorylation site on the $70-k D a$ subunit of neurofilaments. Early turnover during axonal transport. $J$ Biol Chem 1991;266:18861-7

Suh PG, Ryu SH, Moon KH, Suh HW, Rhee SG. Inositol phospholipid-specific phospholipase C: complete cDNA and protein sequences and sequence homology to tyrosine kinase-related oncogene products. Proc Natl Acad Sci USA 1988;85:5419-23

Watson DF, Nachtman FN, Kuncl RW, Griffin JW. Altered neurofilament phosphorylation and beta tubulin isotypes in Charcot-Marie-Tooth disease type 1. Neurology 1994;44: 2383-7

Xu Z, Cork LC, Griffin JW, Cleveland DW. Increased 
expression of neurofilament subunit NF-L produces morphological alterations that resemble the pathology of human motor neuron disease. Cell 1993;73:23-33

Yates DM, Manser C, De Vos KJ, Shaw CE, McLoughlin DM,
Miller CC. Neurofilament subunit (NFL) head domain phosphorylation regulates axonal transport of neurofilaments. Eur J Cell Biol 2009;88:193-202 